\title{
Fraud Detection in E-Commerce Using Machine Learning
}

\author{
Muhammad Ahsan Saeed ${ }^{1}$, Farrukh Yousaf ${ }^{1}$, Osama Bin Khalid ${ }^{1}$, Mushhad Gilani ${ }^{1}$, Qamar Nawaz ${ }^{2}$, \\ Isma Hamid $^{3}$ \\ ${ }^{1}$ University Institute of Information Technology, PMAS Arid Agriculture University Rawalpindi, Pakistan \\ ${ }^{2}$ Department of Computer Science, University of Agriculture Faisalabad, Pakistan \\ ${ }^{3}$ Department of Computer Science, National Textile University, Faisalabad, Pakistan
}

\begin{abstract}
Customers rely heavily on decisions to purchase products either on commerce sites or in online retail outlets. Since these reviews are the game changers for success or failure in product marketing, reviews are used for positive or negative ideas. Improper reviews may also be referred to as false / fraudulent reviews or spam comments or false reviews. To downgrade or advance the item, resentful audits or phony surveys, which are tricky, are posted in the web-based business site. This outcome will prompt possible monetary misfortunes or bigger measure of development in business. So, the proposed system is design and developed in such way that it will detect fake, false and spam reviews for fraud detection using machine learning approaches like Sentiment Analysis, Support Vector Machine (SVM), Decision Tree algorithm, and N-gram model.
\end{abstract}

Key words: Review deviation Reputation systems, Sentiment Analysis, Fake review observation; Spam Detection

\section{INTRODUCTION}

In today's digital world the idea of spams and frauds have become a threat to both customers and companies. Identifying the fake reviews is a complex and difficult task. The fraudulent reviewers are often paid to write these reviews. Because of this, it is the difficult job for the average customer to distinguish fake reviews from real ones, by looking at each review. In addition, to support the sale of goods a few businesses submit positive reviews of their products to influence customer purchasing practices. Because of the uplifted degree of competition in the business, it has become a need for every organization to maintain its esteem and height on the lookout. As a result, splitting automated reviews becomes an important task for customers and businesses. Subsequently, this article proposes to create a machine learning-based framework for identifying spam and fake reviews and non-reviews.

There has been an exciting change in the way people share and express their opinion and credit has gone completely to the social web. Client-generated content is a term used to display something donated by web customers (rather than something provided by site owners). Client created content contains a large amount of data which can be a very bad cause for various other applications if misused.

According to the proposed research, certain systems were considered that supports different attributes and domains like few are used for detection of fraudulent credit card, this is also important because in case of fraudulent credit card heavy financial loss can be caused. And few only detects the fake and fraudulent reviews, but this proposed methodology not only intends to detect fraudulent reviews but also to remove them from the database. This proposed e-commerce web application gets reviews as the inputs from the users, stores them in the database and then proposed methods are used to detect the spammers. In order to perform the task, firstly it is needed to check either a customer is logged in or not. Secondly, check that either a customer bought the product or not. Thirdly, check the credibility of the reviewer. To do this, reviewers Medium Access Control (MAC) address. Internet Protocol (IP) address, location, is accessed. Location and IP addresses can be changed easily using VPNs so there is a need to detect VPNs as well. There is one easy way to do that is to use MAC address because it can't be easily changed. MAC address basically comprises of unique number which is utilized to track a device in a network. It facilitates us with a secure way to locate senders or receivers in the network and helps avoid unwanted network access.

Purpose of proposed approach is to ascertain all the un-authentically fake or spam comments associated to any product that may be causing a high damage on the productivity of seller and additionally manipulating the perspective of consumers about the product. Spam Reviews numerous reviews given by a user for the same product are considered fake. Not necessarily, a loyal customer may review every time the product is bought. The main problem is that all the reviews posted on the website may not be real or genuine.

\section{LITERATURE REVIEW}

In the text, spam reviews are divided into three groups [1].

(1) Untruthful Reviews - a major part of this paper,

(2) Reviews on trademarks - where opinions are solely concerned with the product or the seller of the product and fail to review the product. 
(3) Non-Reviews - those reviews or updates containing text or unrelated ads.

The first phase, the revised review, is of great concern as it undermines the integrity of the online review system. Identifying a type of spam review for a particular type is as challenging, if not impossible, to distinguish between false reviews and self-study. To show the difficulty of this task.

\subsection{Robust Algorithm:}

Amazon has developed a robust algorithm to detect fake reviews, that can be both positive or negative [2]. Fake reviews are positive when bought by the seller and can be negative when bought by the competitors to drop the rating. So, it's a complex matrix to detect fake reviews which Amazon is trying to overcome with the help of technology and the team of professionals to manually monitor. Overall, the approach of Amazon is good but is more time consuming by manually detecting the fake reviews.

\subsection{Fake Review Fraud Detection using Data Mining:}

Another approach towards fake reviews detection is data mining. While it is a good way but it also has restrictions and drawbacks [3].

1. Violates user privacy: It is a well-known fact that data mining collects information about people using certain market-based strategies and information technology.

2. Additional irrelevant information.

3. Misuse of information.

\subsection{Yelp filtering Algorithm:}

Yelp is the largest and most popular online review site that filters untrue or suspicious reviews. It uses various filtering algorithm in order to find out fake reviews. After studying a lot about yelp, it was decided that machine learning approach is always a better approach towards fake and spam reviews detection.

Fake Review Detection and classification and analysis of real and Pseudo reviews in false review analysis and real false review to understand the psyche of false reviewers to produce data sets that can provide high accuracy in detection using supervised learning. Kl-divergence method is used to study data sets behavioral features are used along with n-gram features to check dataset of AMT. AMT's generated dataset was not found as representative of fake reviews and furthermore it was found that behavioral features alone give good results accuracy [4-7].

Exploiting Product Related Review Features for Fake Review Detection exploits product-related review features to detect false review acquisition A convolutional neural network model is suggested to integrate a product with a brand name. For maximum flexibility, the wrap-up strategy is used to incorporate a network model bag with two efficient dividers. Various types of tests were performed to evaluate the performance of the suggested model. Another missing feature can be found in the future that can help you find accurate information [2].

Fake review detection using data mining is the study whose objective was to solve the fake reviews problem by using different data mining techniques and explore the weaknesses and strength in data mining techniques. For this study supervised approach was made to detect the fake reviews which includes Support Vector Machine (SVM), Multinomial Naive Bayes (MNB) \& Multilayer Perceptron in this research, the authors took different approaches for spam review detection. they started with supervised method, then tried with semi-supervised method and finally, used a fully unsupervised method for spam review detection. First, they used supervised approach that requires large scale of datasets then they used semi-supervised which depends a lot on graphs. It was suggested by the authors that in future other methods for validation of Words Basket Analysis (WBA) can be proposed. One suggestion for this purpose is to manually label the fake and real reviews this will help in reducing the size of datasets, then the performance of WBA approach can be improved and for labeling the truthful and deceptive reviews a behavioral approach can be employed [3],

Spam Review Detection Techniques: Systematic Literature Review A study in which researchers conduct a comprehensive review of existing studies on the availability of spam reviews using the Systematic Literature Review (SLR.) In total, 76 existing studies are reviewed and analyzed. Researchers evaluated studies based on how features are extracted from review data sets and the different methods and techniques used to solve spam detection problem detection. This study has shown that the success of any spam retrieval review method depends. Feature releases depend on the update database, and the accuracy of spam detection methods depends on the choice of feature engineering method. Therefore, in the successful use of the spam review acquisition model and achieve better accuracy, these factors need to be considered in conjunction. To the knowledge of the researchers, this is the first complete review of existing studies in the field of spam review for the use of the SLR process. This study presented a systematic review of the literature on the field of spam review findings and highlighted the contributions of recent research in the form of various engineering methods, methods of detecting spam reviews, and various measures used in performance testing. To bring out direct pragmatic evidence, this work organized a review process, focused on a search query, raised research questions, selected papers from reputable publishers, applied formal submissions and a study assessment process. The main advantage of this study is that, to our knowledge, this is the first attempt to integrate all available spatial reviews of spam reviews using the SLR method. In addition, the release of this study may assist in further research in the field of spam review [8]. 
An Empirical Study on Detecting Fake Reviews Using Machine Learning Strategies, this paper receives false reviews using machine learning methods many ways to analyze the data of movie reviews and introduce the algorithms for dividing the emotions and guiding the learning used in this work with stops and non-stop word methods [9]. Algorithms are emotionally divided by the Install tool, which is used to separate movie update databases into non-fiction updates. Finally, in the future this can be applied to different commerce websites like the amazon e bay dataset or a different movie review database and use a variety of options. This method does not apply to default commerce websites. Because its algo produces manual results [10].

After thoroughly viewing different research papers on fraud detections. We came to a point where we believe that approaching techniques to deal with fraud detections are many and different algorithms for fraud detections are designed. But keeping the world need in mind there was a missing factor related to this and that is Machine Learning. An approach that covers almost every aspect towards fraud detection handling that's why the idea that we have proposed are much more up to date and much more efficient while dealing with fraud detection. Table1 illustrates the comparison of different research papers.

Table 1: Summary of literature comparison

\begin{tabular}{|l|l|l|l|l|l|}
\hline $\begin{array}{l}\text { Research } \\
\text { Article }\end{array}$ & $\begin{array}{l}\text { IP } \\
\text { Addres } \\
\text { S }\end{array}$ & $\begin{array}{l}\text { Mac } \\
\text { Addre } \\
\text { ss }\end{array}$ & Location & $\begin{array}{l}\text { Gmail } \\
\text { ID }\end{array}$ & $\begin{array}{l}\text { Spam } \\
\text { word } \\
\text { detect } \\
\text { ion }\end{array}$ \\
\hline $\begin{array}{l}\text { Mukherjee } \\
\text { et al }\end{array}$ & $\mathbf{x}$ & $\mathbf{x}$ & $\mathbf{x}$ & $\mathbf{x}$ & $\checkmark$ \\
\hline Sun et al & $\mathbf{x}$ & $\mathbf{x}$ & $\mathbf{x}$ & $\mathbf{x}$ & $\checkmark$ \\
\hline $\begin{array}{l}\text { Hossain } \text { et } \\
\text { al }\end{array}$ & $\mathbf{x}$ & $\mathbf{x}$ & $\mathbf{x}$ & $\mathbf{x}$ & $\checkmark$ \\
\hline Mirza et al & $\mathbf{x}$ & $\mathbf{x}$ & $\mathbf{x}$ & $\mathbf{x}$ & $\checkmark$ \\
\hline $\begin{array}{l}\text { Elmurngi } \\
\text { et al }\end{array}$ & $\mathbf{x}$ & $\mathbf{x}$ & $\mathbf{x}$ & $\mathbf{x}$ & $\checkmark$ \\
\hline Bajaj et al & $\checkmark$ & $\mathbf{x}$ & $\checkmark$ & $\checkmark$ & $\mathbf{x}$ \\
\hline
\end{tabular}

\section{Problem Statement}

The proposed software solution will help the user to determine the fake unauthentic and spam reviews of any product. It always has been an immense problem that all the buyers face when buying stuffs from e-commerce webstores. Fake and spam comments most of the time manipulate the buyers which deplorably impact on the productivity of the seller at astronomically immense level. With all of our research we have ascertain that fake reviews are still an immensely colossal issue for all e-commerce community. With proposed software store owners will be able to detect these fake reviews and withdraw them from the database.

As discussed above, this system is being developed to mainly target the unauthentically spurious and spam reviews in order to make the e-commerce environment better. People relish to read the reviews before they made up their minds to buy anything from webstores. By detecting fake reviews and eliminating them will lead us to the point where buyers will not be further manipulated by any of this unauthentic and spurious reviews stuff. Buying and selling of products will get more frequent and facile.

According to Literature review, it is ascertained that system like this subsists but with the different attributes and domain. Some of them are fraud detection of credit cards and some are predicated on fake reviews detection, but we are intended to engender a product that gives us access to not just find those reviews out but withal to efface them from database. This will let us to maintain the webstores and keep a check and balance of reviews.

\section{PROPOSED METHOD}

Usually, it is noticed that an original buyer will review on the quality of the service or product only once until or unless he or she wants to respond to other customers or deliberately wants to misguide others by hyper or fake reviews.

The attributes of spam reviews \& non-spam as discussed in the next section in detail are used to build the database. A web application has been created that captures user reviews, which stores data in a database and detects spam according to the proposed method. Customer identification is tracked using its login email, location and IP address of its device and Mac address. The process as discussed in Figure.1

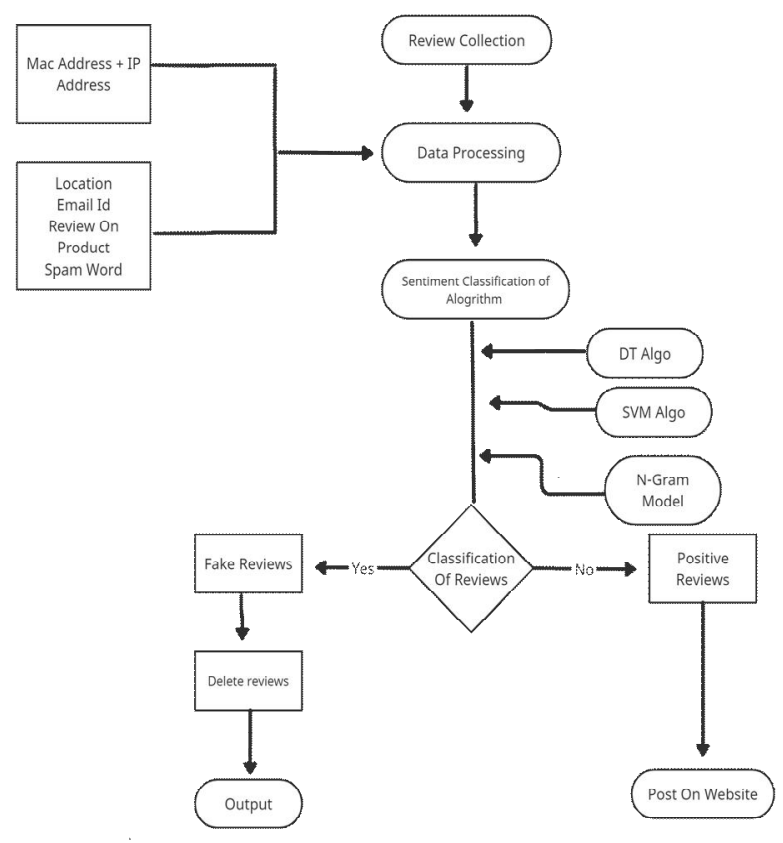

Figure 1: Flow of proposed method 


\section{i. MAC ADDRESS}

A person who is came to do fraud activity can change his IP address by switching his network. Also, the device location can be changed by using VPN. So, to overcome these main above mentioned problems mac address is used. When a user doing different types of fraud reviews, proposed system gets his mac address because it can't be replaced. We can stop his fraud reviews by blocking his mac address he can't be able to detect the online website.

\section{ii. IP Address}

Customers can use any devices to send incorrect reviews. However, capturing the device's IP address can help identify the device in particular. Our proposed version determines the times when a user is allowed to send online reviews from his device to a single product. A true customer will not attempt to post multiple reviews for the same PRODUCT OVER AND OVER again. How to stop spam you can mislead users, which is very annoying. But spam can change their IP address by changing their network or other source.

\section{iii. EMAIL ID}

Every website allows a customer to access their services and allow transactions only after verifying their ownership, i.e., the user must create an account on that website. One component of the information required by each online service website is the customer email id. On proposed web application, the buyer may send only 1 review to a specific product by the email provided. In the event that a customer logs into an email website for example xyz@gmail.com, and the next time they try to log in using xy.z@gmail.com, then existing E-commerce websites treat them as two separate accounts or customers. two different. But in reality, these IDs are the same in relation to email. Therefore, it is easier for spammers to sign in with different accounts and send multiple updates with different identities all the time. To avoid this, we have suggested the removal of names and compare whether the IDs are the same or different. A better idea would be to let the email handle authenticity and instead of having a separate subscription, simply allow the customer to use the website using his or her email. The proposed web application implements both methods. But what if spammer creates another email address? Although it will be difficult to get a new ID every time you post a review but yes, it is possible. To combat this attack, we can track the IP address of the device.

\section{iv. LOCATION}

Another feature used is the customer's location. To determine the person's location, it is just needed to find the persons longitude and latitude and where the person provides reviews to the product. When the location is found, we look at how many reviews of the same product are offered from the same location. A mock reviewer trying to increase or decrease a product will post more than one review to affect its rating which may also adversely affect another customer's purchase decision. But location can also be changed using a VPN.

\section{v. SPAM WORD}

The spam dictionary is used to find unrelated reviews. We have taken inspiration from previous studies to find the spam word 'buy direct', 'money back', 'the offer'. These spam words play a vital role in misleading consumers. Database is created that store these types of spam words using $\mathrm{N}$-gram model \& Jaccard coefficient. The flow of buying and review process of products can be seen in Figure 2 and Figure 3.

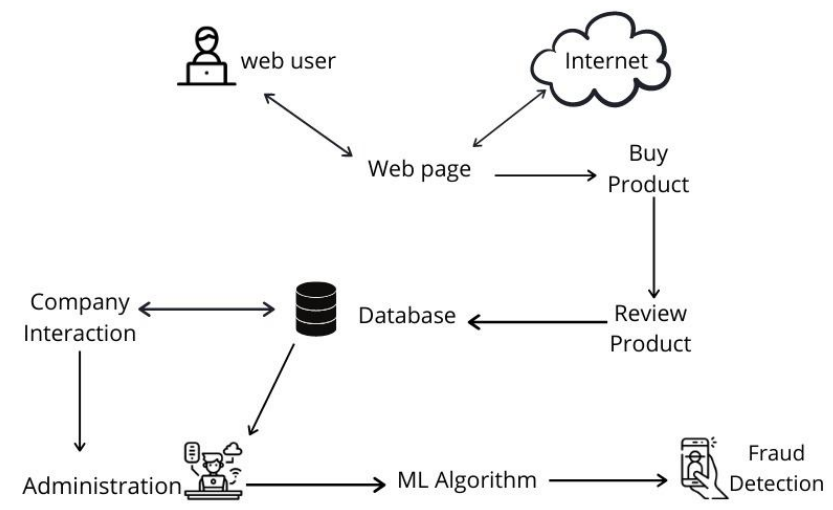

Figure 2 : Flow of review and buying of products
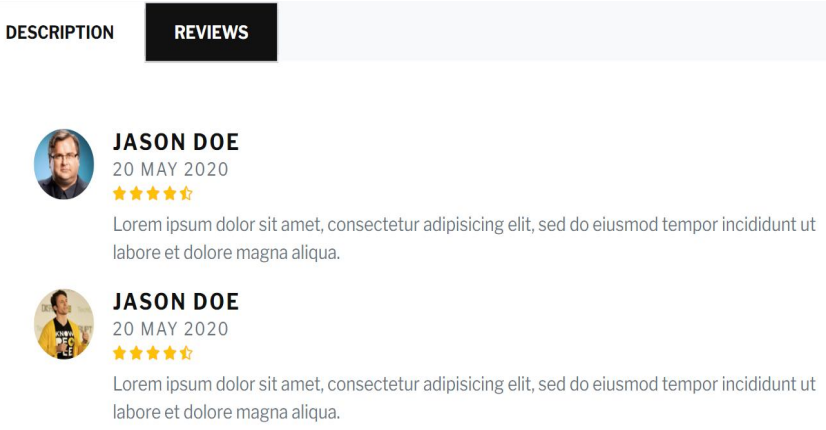

Figure 3: Reviews of products

\section{Conclusion \& Result}

In this work it was the aimed to propose a new way of finding fake reviews that affect the buying behaviors of customers. Our purposed strategy can cover a few attributes of a reviewer, in this way, keeping a remarkable personality for every client. It is adequately ready to perceive spam exercises under certain presumptions. The preliminary experiments have shown promising results.

After viewing variety of research on fraud detection, it was found that work has been done on spam words utilizing different techniques, but still detection was destitute in some manners which involves other aspects like IP addresses, MAC addresses and Email accounts additionally Machine Learning approaches were missing and very less work has been done till now utilizing these approaches. Some researchers suggested the utilization of IP addresses was found but still it was the destitute in mac addresses so proposed approach covered all these aspects which involves IP addresses, mac addresses, 
Table 2: Summary and classification of Reviews on same product

\begin{tabular}{|c|c|c|c|c|c|c|c|c|}
\hline $\begin{array}{l}\text { Sr. } \\
\text { No }\end{array}$ & Reviews & ID & Mac Address & IP Address & Latitude & $\begin{array}{l}\text { Longit } \\
\text { ude }\end{array}$ & $\begin{array}{c}\text { Spam } \\
\text { word }\end{array}$ & impact \\
\hline 1 & Great product & $\begin{array}{c}\text { Ahsa164@gmail. } \\
\text { com }\end{array}$ & $\begin{array}{c}\text { E4:A7;C5:A4:CF:D } \\
\text { D }\end{array}$ & $\begin{array}{c}192.168 .64 . \\
1\end{array}$ & 28.65289 & $\begin{array}{c}34.890 \\
87\end{array}$ & $\overline{--}$ & non-spam \\
\hline 2 & $\begin{array}{l}\text { Nice product } \\
\text { and quality }\end{array}$ & $\begin{array}{c}\text { Ahsa164gmail.co } \\
\text { m }\end{array}$ & $\begin{array}{c}\text { E4:A7;C5:A4:CF:D } \\
\text { D }\end{array}$ & $\begin{array}{c}192.168 .65 . \\
2\end{array}$ & 28.65289 & $\begin{array}{c}34.890 \\
87\end{array}$ & $\begin{array}{c}\text { Money } \\
\text { back }\end{array}$ & spam \\
\hline 3 & $\begin{array}{l}\text { Nice features, } \\
\text { happy to buy this } \\
\text { and get cash } \\
\text { back as bonus }\end{array}$ & ali164gmail.com & 98:40;C5:A4:CF:2D & $\begin{array}{c}192.168 .67 . \\
1\end{array}$ & 18.65289 & $\begin{array}{c}23.890 \\
87\end{array}$ & $\begin{array}{l}\text { Cash } \\
\text { bonus }\end{array}$ & spam \\
\hline 4 & $\begin{array}{l}\text { Low quality. bad } \\
\text { product. Want } \\
\text { my money back. }\end{array}$ & $\begin{array}{c}\text { Zahid1122@gmai } \\
\text { 1.com }\end{array}$ & 09:0A;C5:A9:7F:9B & $\begin{array}{c}192.168 .69 . \\
3\end{array}$ & 28.65289 & $\begin{array}{c}37.890 \\
87\end{array}$ & $\begin{array}{l}\text { Money } \\
\text { back }\end{array}$ & spam \\
\hline 5 & $\begin{array}{l}\text { Seller doesn't } \\
\text { send product as } \\
\text { described. Want } \\
\text { my money back }\end{array}$ & $\begin{array}{l}\text { Zahid11223@gm } \\
\text { ail.com }\end{array}$ & 09:0A:C5:A3:4F:9B & $\begin{array}{c}192.168 .64 . \\
1\end{array}$ & 28.65289 & $\begin{array}{c}34.890 \\
87\end{array}$ & $\begin{array}{l}\text { Money } \\
\text { back }\end{array}$ & spam \\
\hline 6 & $\begin{array}{l}\text { Just received my } \\
\text { product }\end{array}$ & abc@gmail.com & A4:7F;C5:A4:CF:2F & $\begin{array}{c}192.116 .64 . \\
1\end{array}$ & 28.65289 & $\begin{array}{c}34.890 \\
87\end{array}$ & -- & Non-spam \\
\hline 7 & $\begin{array}{l}\text { Amazing } \\
\text { product }\end{array}$ & alia@gmail.com & AF:7C:C5:88:CF:9B & $\begin{array}{c}192.168 .79 . \\
1\end{array}$ & 37.65289 & $\begin{array}{c}57.890 \\
87\end{array}$ & -- & Non-spam \\
\hline 8 & $\begin{array}{l}\text { Not up to the } \\
\text { mark }\end{array}$ & zoha@gmail.com & $\begin{array}{c}\text { E4:A7;C5:A4:CF:D } \\
\text { D }\end{array}$ & $\begin{array}{c}192.168 .75 . \\
1\end{array}$ & 67.65289 & $\begin{array}{c}78.890 \\
87\end{array}$ & -- & Non-spam \\
\hline
\end{tabular}

Email account utilizing a Machine Learning technique to make the detection more efficient than it was ever before and more facile in case of different scarcely frauds.

The only one time a customer can post a review from given email, with specific MAC address, and from particular location. The Purposed method used \& tested on a set of 90 reviews, and it shows accuracy up to $75 \%$.

In Figure 3 \& Table 2 results of our purposed method are presented. Review 2 having same email address and same MAC address as that of review 1, therefore, it has been considered spam because user repeatedly commented in the favor of product. In Review $4 \& 5$ there are spam words like, "I Want my money back. Also, the device which the user is using has same MAC Address, so it is considered spam.

\section{ACKNOWLEDGEMENT}

It is hereby to apprise you that this research is not copied from anywhere and doesn't contain any plagiarism. We are thankful to our PMAS Arid Agriculture University UIIT campus for giving us this astounding opportunity of inscribing a research paper. We thank Dr. Yasir Hafeez for his assistance and guidance and additionally this research would never have been possible without the great supervision of Dr. Mushhad Gilani who guided us in every single details. Availed us in all manners in order to get this research done.

\section{REFERENCES}

[1] H. Ahmed, I. Traore, and S. Saad, "Detecting opinion spams and fake news using text classification," Security and Privacy, vol. 1, no. 1, p. e9, 2018.

[2] C. Sun, Q. Du, and G. Tian, "Exploiting product related review features for fake review detection," Mathematical Problems in Engineering, vol. 2016, 2016.

[3] M. F. Hossain, "Fake review detection using data mining," 2019.

[4] A. Mukherjee, V. Venkataraman, B. Liu, and N. Glance, "Fake review detection: Classification and analysis of real and pseudo reviews," UIC-CS-03-2013. Technical Report, 2013.

[5] H. B. Abdalla, J. Lin, G. Li, S. M. M. J. I. J. o. I. Gilani, and E. Engineering, "NoSQL: Confidential on Data Security and Data Management by Using a Mobile Application," vol. 6, no. 2, p. 84, 2016.

[6] A. Mahmood, S. M. M. Gilani, M. J. Iqbal, Z. Haider, and S. J. T. J. Daud, "Analysis and Evaluation of Secure Solutions for Terrestrial Networks," vol. 24, no. 04, pp. 63-71, 2019.

[7] A. Thakur, B. Shaikh, V. Jain, and A. Magar, "INTERNATIONAL JOURNAL OF 
ENGINEERING SCIENCES \& RESEARCH TECHNOLOGY CREDIT CARD FRAUD DETECTION USING HIDDEN MARKOV MODEL AND ENHANCED SECURITY FEATURES."

[8] N. Hussain, H. Turab Mirza, G. Rasool, I. Hussain, and M. Kaleem, "Spam review detection techniques: A systematic literature review," Applied Sciences, vol. 9, no. 5, p. 987, 2019.

[9] L. Nawaz and Q. Nawaz "An Improved Methodology for Data Hiding In Images Using Haar Transformed, LSB Replacement Method and Modified PVDMF 1," International Journal of Advanced Trends in Computer Science and Engineering, vol. 10, no. 3, pp. 1690-1699, 2021.

[10] E. Elmurngi and A. Gherbi, "An empirical study on detecting fake reviews using machine learning techniques," in 2017 seventh international conference on innovative computing technology (INTECH), 2017, pp. 107-114: IEEE. 